EPJ Web of Conferences 78, 07002 (2014)

DOI: $10.1051 /$ epjconf/20147807002

(C) Owned by the authors, published by EDP Sciences, 2014

\title{
The unreasonable effectiveness of experiments in constraining nova nucleosynthesis
}

\author{
Anuj Parikh1,2,a \\ ${ }^{1}$ Departament de Física i Enginyeria Nuclear, Universitat Politècnica de Catalunya, EUETIB, E-08036 \\ Barcelona, Spain \\ ${ }^{2}$ Institut d'Estudis Espacials de Catalunya (IEEC), E-08034 Barcelona, Spain
}

\begin{abstract}
Classical nova explosions arise from thermonuclear ignition in the envelopes of accreting white dwarfs in close binary star systems. Detailed observations of novae have stimulated numerous studies in theoretical astrophysics and experimental nuclear physics. These phenomena are unusual in nuclear astrophysics because most of the thermonuclear reaction rates thought to be involved are constrained by experimental measurements. This situation allows for rather precise statements to be made about which measurements are still necessary to improve the nuclear physics input to astrophysical models. We briefly discuss desired measurements in these environments with an emphasis on recent experimental progress made to better determine key rates.
\end{abstract}

\section{Introduction}

A classical nova explosion arises from a thermonuclear runaway in a shell of hydrogen-rich material accreted onto the surface of a white dwarf star in a close binary star system (for reviews, see e.g., Refs. [1-3]). As accretion proceeds, the envelope is gradually compressed and becomes degenerate. The temperature of the envelope increases, creating conditions favorable to the ignition of the accreted fuel through nuclear reactions. These reactions, once initiated, drive further reactions, leading to the thermonuclear runaway and the corresponding explosion. Several hundred Galactic novae have been discovered to date, with roughly five events discovered per year. Light curves for these events peak at $\approx 10^{4}-10^{5}$ times the solar luminosity and persist for intervals of $\approx$ days to several months. A typical nova explosion will eject $\approx 10^{-4}-10^{-5}$ solar masses of material into the interstellar medium.

Spectroscopy of the ejecta has revealed that most novae show overabundances relative to solar of carbon, nitrogen and oxygen with $\approx 1 / 3$ also showing an overabundance of neon. Traces of heavier elements up to the calcium region are also seen. This observed enhancement in material heavier than helium is thought to occur both through nucleosynthesis during the nova explosion and through mixing of the accreted matter with material from the white dwarf itself. Explaining spectroscopic observations of nova ejecta therefore requires not only knowledge of this mixing mechanism (see e.g., Ref. [4] for recent advances) but also knowledge of the nuclear reaction rates involved in the explosion.

\footnotetext{
a e-mail: anuj.r.parikh@upc.edu
} 


\section{Stellar models and nucleosynthesis}

State-of-the-art nucleosynthesis calculations for novae currently rely exclusively on 1-D hydrodynamic models (e.g., Refs. [5-11]). The underlying assumption in these models is, obviously, spherical symmetry, where the explosion is modeled as occurring uniformly and simultaneously over spherical shells. In contrast, these thermonuclear runaways are expected to originate from point-like ignitions. As such, multidimensional hydrodynamic simulations (see e.g., Refs. [4, 12-17]) should be used for improved nucleosynthesis predictions when sufficient computational power is available to model all relevant details of the explosion. To date, multidimensional models have only followed the evolution of a nova over only a very small fraction of the overall time associated with the event (e.g., $\sim 1000 \mathrm{~s}$ near the peak temperature, to be compared with the duration of the accretion stage $\sim 10^{5} \mathrm{yr}$ ).

The observed (elemental) composition of ejecta from novae is in broad agreement with predictions from current (1-D) models. Measurements of the relative abundances of different isotopes in nova ejecta could further improve model constraints. Such information could be provided through the detection of $\gamma$-rays from the decay of radioisotopes produced during the explosion, or through measurements of presolar grains - microscopic grains embedded within primitive meteorites. Unfortunately, in the former case, only upper limits on nuclear $\gamma$-ray emission from novae have been obtained to date (all of which are fully compatible with theoretical predictions); and in the latter case, only a handful of measured grains exhibit signs of nova nucleosynthesis [18] - and even these may also be consistent with origin in type II supernovae [19, 20].

Because nucleosynthesis in classical nova explosions is normally restricted to nuclei near the valley of stability and to masses less than $A \approx 40$, many of the thermonuclear reaction rates involved have been constrained using experimental information [21]. This has (not "unreasonably"!) improved the precision of nucleosynthesis predictions from models. Studies have identified uncertainties in the rates of the ${ }^{18} \mathrm{~F}(p, \alpha){ }^{15} \mathrm{O},{ }^{25} \mathrm{Al}(p, \gamma){ }^{26} \mathrm{Si},{ }^{30} \mathrm{P}(p, \gamma){ }^{31} \mathrm{~S}$ and ${ }^{33} \mathrm{~S}(p, \gamma){ }^{34} \mathrm{Cl}$ reactions as dominant contributors to remaining uncertainties in nova nucleosynthesis, and many recent measurements have focused on better determining these rates (see e.g., Ref. [3] for a review). We briefly discuss recent progress below. Additional nova simulations are encouraged to confirm the relative robustness of nova nucleosynthesis to current nuclear physics uncertanities.

\section{Experimental nuclear physics}

If a reaction rate is dominated by isolated and narrow resonances, as is the case for many reactions involved in novae, we may use the Breit-Wigner cross-section formula to write the reaction rate per particle pair $\langle\sigma v\rangle$ at a temperature $T$ as [22]

$$
\langle\sigma v\rangle=\left(\frac{2 \pi}{\mu k T}\right)^{3 / 2} \hbar^{2} \sum_{i} e^{-E_{R, i} / k T}(\omega \gamma)_{i}
$$

where $\mu$ is the reduced mass of the reactants and the $E_{R, i}$ are resonance energies. The resonance strength $\omega \gamma$ can be expressed as

$$
\omega \gamma=\frac{2 J_{R}+1}{\left(2 J_{1}+1\right)\left(2 J_{2}+1\right)} \cdot \frac{\Gamma_{a} \Gamma_{b}}{\Gamma_{t o t}}
$$

where $J_{R}, J_{1}$, and $J_{2}$ are the spins of the resonance and the reactants, and $\Gamma_{t o t}, \Gamma_{a}$ and $\Gamma_{b}$ are the total width and partial widths of the entrance and exit channels of the resonance. The sum in Eq. (1) allows for the contributions of all resonant states through which the reaction may proceed at the chosen temperature. 
The ${ }^{18} \mathrm{~F}(p, \alpha){ }^{15} \mathrm{O}$ and ${ }^{25} \mathrm{Al}(p, \gamma){ }^{26} \mathrm{Si}$ reaction rates are of interest as they affect the yields of the potentially observable radioisotopes ${ }^{18} \mathrm{~F}$ and ${ }^{26} \mathrm{Al}$ produced in nova explosions. Fig. 1 shows ${ }^{18} \mathrm{~F}(p, \alpha)$ rates calculated in Ref. [23]. When used with 1-D hydrodynamic nova models, these rates result in ${ }^{18} \mathrm{~F}$ yields that differ by a factor of $\approx 2[23]$, indicating the need for a measurement of the strength of the $48 \mathrm{keV}$ resonance. On the other hand, recent work to determine and test the impact of resonance parameters used to calculate the ${ }^{25} \mathrm{Al}(p, \gamma)^{26} \mathrm{Si}$ rate $[24,25]$ indicates that the predicted nova yield of ${ }^{26} \mathrm{Al}$ is essentially independent of the uncertainty in this rate.

Uncertainties in the ${ }^{30} \mathrm{P}(p, \gamma){ }^{31} \mathrm{~S}$ and ${ }^{33} \mathrm{~S}(p, \gamma){ }^{34} \mathrm{Cl}$ reaction rates affect the nova yields of species between Si and Ca. Figs. 2 and 4 show rates for these two reactions as calculated in Refs. [26, 28]. When used with 1-D hydrodynamic nova models, these rates result in yields that differ by as much as a factor of $\approx 8$ (see Figs. 3 and 5). The uncertainties in these rates arise from the unknown strengths of low-energy proton-threshold resonances (i.e., below $\mathrm{E}_{x}\left({ }^{31} \mathrm{~S}\right)=6.7 \mathrm{MeV}$ for ${ }^{30} \mathrm{P}(p, \gamma)$, and below $\mathrm{E}_{x}\left({ }^{34} \mathrm{Cl}\right)=5.4 \mathrm{MeV}$ for $\left.{ }^{33} \mathrm{~S}(p, \gamma)\right)$; as well, even $J^{\pi}$ values for relevant states have been debated [26, 29].

\section{Outlook}

When feasible, modelers should work to evolve multidimensional hydrodynamic nova model calculations from the accretion stage through the explosion and ejection stages. In the meantime, new comprehensive studies of the impact of current nuclear physics uncertainties on nucleosynthesis predictions are needed, ideally using 1-D hydrodynamic models. Experimentalists should build upon recent accomplishments to fully characterize the rates of e.g., the ${ }^{18} \mathrm{~F}(p, \alpha){ }^{15} \mathrm{O}$ and ${ }^{30} \mathrm{P}(p, \gamma){ }^{31} \mathrm{~S}$ reactions for nova explosions. Additional constraints on nucleosynthesis predictions from novae (as well as implications of available constraints from elemental observations [30, 31]) should be explored further. This may include the search for additional signatures that may help to distinguish between presolar grains of nova and supernova origin.

\section{Acknowledgements}

We thank E. Wigner for his many contributions to modern physics and for inspiring the misleading title of this short contribution.

\section{References}

[1] M. F. Bode and A. Evans, Classical Novae, 2nd ed., eds. M. F. Bode, A. Evans (Cambridge Astrophysics Series, No. 43, Cambridge Univ. Press, 2008).

[2] J. José and M. Hernanz, J. Phys. G: Nucl. Part. Phys. 34, 431 (2007).

[3] A. Parikh, J. José and G. Sala, AIP Advances 4, 041002 (2014).

[4] J. Casanova, et al., Nature 478, 490 (2011).

[5] D. Prialnik and A. Kovetz, Astrophys. J. 445, 789 (1995).

[6] J. José and M. Hernanz, Astrophys. J. 494, 680 (1998).

[7] S. Starrfield, et al., MNRAS 296, 502 (1998).

[8] O. Yaron, et al., Astrophys. J. 623, 398 (2005).

[9] S. Starrfield, et al., Astrophys. J. 692, 1532 (2009).

[10] M. M. Shara, et al., Astrophys. J. 725, 831 (2010).

[11] P.A. Denissenkov, et al., Astrophys. J. 762, 8 (2013). 
[12] A. Shankar, D. Arnett, and B. A. Fryxell, Astrophys. J. 394, L13 (1992).

[13] A. Shankar and D. Arnett, Astrophys. J. 433, 216 (1994).

[14] S. A. Glasner and E. Livne, Astrophys. J. 445, L149 (1995).

[15] S. A. Glasner, E. Livne, and J. W. Truran, Astrophys. J. 475, 754 (1997).

[16] A. Kercek, W. Hillebrandt, and J. W. Truran, Astron. Astrophys. 337, 379 (1998); and ibid., 345, 831 (1999).

[17] J. Casanova, et al., Astron. Astrophys. 513, L5 (2010).

[18] J. José, et al., Astrophys. J. 612, 414 (2004).

[19] L. R. Nittler and P. Hoppe, Astrophys. J. 631, L89 (2005).

[20] J. José and M. Hernanz, Meteoritics Plan. Sci. 42, 1135 (2007).

[21] C. Iliadis, et al., Nucl. Phys. A841, 21 (2010).

[22] C. Iliadis, Nuclear Physics of Stars (Wiley-VCH, Weinheim, 2007).

[23] A. Laird, et al., Phys. Rev. Lett. 110, 032502 (2013).

[24] M.B. Bennett, et al., Phys. Rev. Lett. 111, 232503 (2013).

[25] A. Parikh and J. José, Phys. Rev. C 88, 048801 (2013).

[26] A. Parikh, et al., Phys. Rev. C 83, 045806 (2011).

[27] J. José, A. Coc, and M. Hernanz, Astrophys. J. 560, 897 (2001).

[28] J. Fallis, et al., Phys. Rev. C 88, 045801 (2013).

[29] D.T. Doherty, et al., Phys. Rev. Lett. 108, 262502 (2012).

[30] L. N. Downen, et al., Astrophys. J. 762, 105 (2013).

[31] K. J. Kelly, et al., Astrophys. J. 777, 130 (2013). 


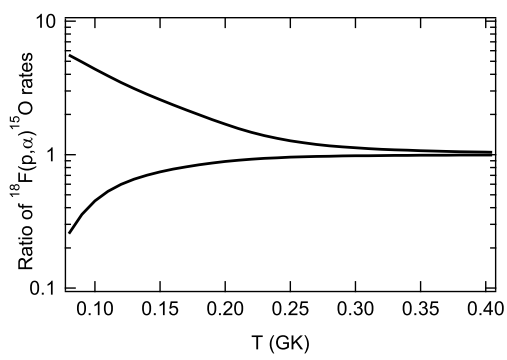

Figure 1. Thermonuclear ${ }^{18} \mathrm{~F}(p, \alpha){ }^{15} \mathrm{O}$ rates over typical nova peak temperatures. Rates calculated in Ref. [23] with (i) zero contribution and (ii) an estimated upper limit to the contribution from the $48 \mathrm{keV}$ resonance are presented, relative to the nominal rate in that work.

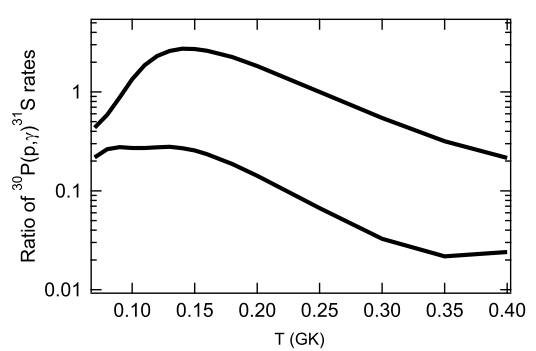

Figure 2. Thermonuclear ${ }^{30} \mathrm{P}(p, \gamma){ }^{31} \mathrm{~S}$ rates over typical nova peak temperatures. Low and high rates calculated in Ref. [26] are presented, relative to a statistical model rate (see Ref. [27]).

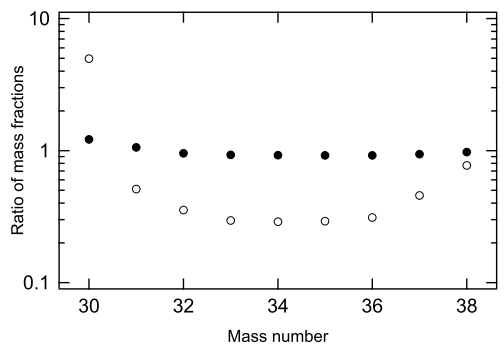

Figure 3. Impact of the ${ }^{30} \mathrm{P}(p, \gamma){ }^{31} \mathrm{~S}$ rates in Fig. 2 on nova nucleosynthesis [26]. Mass fractions calculated using the upper (solid circles) and lower (open circles) rates from Fig. 2, relative to mass fractions calculated using a statistical model rate [27] are presented.

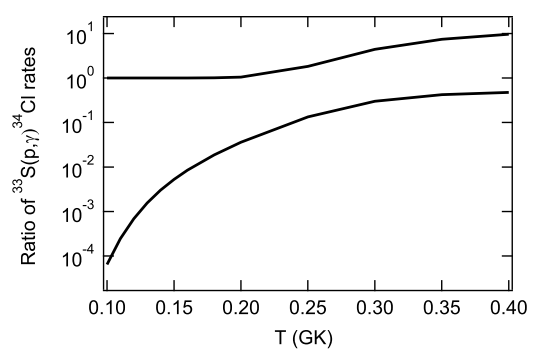

Figure 4. Thermonuclear ${ }^{33} \mathrm{~S}(p, \gamma){ }^{34} \mathrm{Cl}$ rates over typical nova peak temperatures. Rates A and B calculated in Ref. [28], are presented, relative to Rate D in that work.

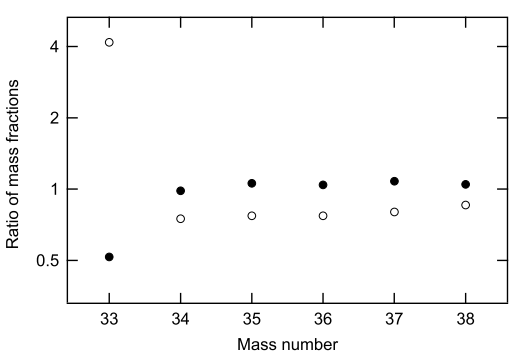

Figure 5. Impact of the ${ }^{33} \mathrm{~S}(p, \gamma){ }^{34} \mathrm{Cl}$ rates in Fig. 4 on nova nucleosynthesis [28]. Mass fractions calculated using the upper (solid circles) and lower (open circles) rates from Fig. 4, relative to mass fractions calculated using Rate D from Ref. [28] are presented. 
